\title{
Multilinguales
}

\section{Le voyage de Chevrillon au Maroc : le monde se lit au pluriel}

$$
\text { رحلة شفريون إلى ألمغرب :العالم يقرأ بصيغة الجمع }
$$

\section{Abdelhak Zerrad}

\section{(2) OpenEdition}

12 Journals

Édition électronique

URL : http://journals.openedition.org/multilinguales/481

DOI : $10.4000 /$ multilinguales.481

ISSN : 2335-1853

Éditeur

Université Abderrahmane Mira - Bejaia

Référence électronique

Abdelhak Zerrad, «Le voyage de Chevrillon au Maroc : le monde se lit au pluriel », Multilinguales [En ligne], 8 | 2017, mis en ligne le 01 juin 2017, consulté le 17 septembre 2019. URL : http:// journals.openedition.org/multilinguales/481; DOI : 10.4000/multilinguales.481

Ce document a été généré automatiquement le 17 septembre 2019.

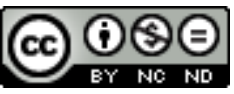

Multilinguales est mise à disposition selon les termes de la Licence Creative Commons Attribution Pas d'Utilisation Commerciale - Pas de Modification 4.0 International 


\title{
Le voyage de Chevrillon au Maroc : le monde se lit au pluriel
}

\author{
رحلة شفريون إلى ألمغرب :العالم يقرأ بصيغة الجمع
}

\author{
Abdelhak Zerrad
}

1 Le récit de voyage, dans sa signification positive, est une forme de méditation sur l'ailleurs et de rencontre avec l'altérité. Ce récit, comme dépositaire de l'expérience de l'horizontalité et de l'hétérogénéité, paraît comme un stimulateur d'interaction et d'échange entre le Moi et l'Autre. En effet, la perception de cet Autre est indissociable de la perception de soi, les deux se complètent et se reflètent immanquablement. Selon Emmanuel Levinas: "l'Autre est posé de façon qu'il ait de moi-même une apparence extérieure. ( (Emmanuel Levinas, 1984 : 344).

2 L'extériorité a attisé la curiosité et l'intérêt des écrivains voyageurs qui se sont posés de nombreuses questions d'ordre ontologique, philosophique et culturel sur la rencontre avec l'altérité. Cette rencontre s'impose comme un principe dynamique et fondamental du voyage.

3 André Chevrillon, au cours de son déplacement au Maroc en 1905, a mis en exergue sa propre vision de l'altérité, une vision animée par l'idéalisation et la considération plutôt que par la confrontation ${ }^{1}$ et la stigmatisation. De ce point de vue, le voyageur prend ses distances vis-à-vis du processus de schématisation de l'Autre par des images qui le caricaturent à l'extrême. Par son approche relativiste qui tend vers la positivité plutôt que vers la négativité, Chevrillon choisit la déconstruction de l'imaginaire occidental représentant l'altérité par des a priori peu fondés qui donnent naissance aux stéréotypes et aux clichés. Aussi, le voyageur, qui se déplace au Maroc dans une époque agitée par le mouvement colonial, refuse-t-il de s'inscrire dans toute approche idéologique infériorisant l'Autre en vue de préparer sa domination. Rejetant ce ton hautain, le voyageur critique acerbement l'idée de l'Occident dominateur et ethnocentriste qui réduit l'altérité à l'infirmité et la condamne à l'inhumanité. De plus, il met en doute la systématicité et la superficialité de la pensée occidentale qui opte pour une approche classificatoire et qui trace la dichotomie entre « eux » et «nous $»^{2}$. 
Une telle hiérarchisation ne pourrait donner lieu qu'à la négation de l'Autre et la peur de l'Autre.

Dans notre étude, nous essaierons de montrer, dans un premier moment, comment le voyage au Maroc développe chez Chevrillon une attitude morale et culturelle fondée essentiellement sur la reconnaissance et le respect de l'Autre dans son altérité, sa typicité et sa relativité. Dans un second moment, nous montrerons comment le voyageur français, croyant à un monde au pluriel, opte pour un exotisme respectueux de la différence. Cette éthique relationnelle basée sur le respect de la diversité ne pourrait-elle pas être un antidote contre ce que Todorov appelle "un choc des civilisations préfabriqué»? (Edward Saïd, 1980) ${ }^{3}$

\section{Le relativisme culturel : la diversité face à l'adversité}

5 Le relativisme culturel se présente comme un courant de pensée qui préconise la relativité des valeurs, des normes et des codes moraux. Ceci implique la différence et la variation du système culturel d'une société à l'autre et d'une civilisation à l'autre. Si, pour Platon, «la vertu est toujours une et toujours la même» (Michel Malherbe, $2000: 39$ ), Montaigne en revanche pense que : «Chaque nation s'en forme une idée différente » (1994: 38).

6 A l'instar de Montaigne, André Chevrillon dans Un crépuscule d'Islam opte pour le relativisme en manifestant et son respect de la culture marocaine et sa reconnaissance de la différence et de la diversité incarnées par l'altérité. Cette attitude positive constitue un recul critique vis-à-vis de l'européocentrisme qui catégorise et caractérise les groupes extra-européens selon une échelle de valeurs purement occidentale. Ladite attitude constitue également une réaction contre les formes les plus nébuleuses de l'impérialisme culturel qui essentialise les cultures d'une manière axiologique et arbitraire. Chevrillon s'oppose à l'Occident qui cherche à imposer le primat de son éthique et qui se prend pour l'unique modèle civilisationnel. Il s'oppose de même au totalitarisme du vrai véhiculé par cet Occident qui prétend à la supériorité et qui fait preuve de son incapacité à saisir l'altérité dans sa vérité et son essence. Par cette démarche relativiste, le voyageur, dans sa lecture du Maroc, a remis en question la pensée unique et a suggéré en contrepartie une pensée au pluriel ${ }^{4}$, comme l'indique sa mise en valeur de l'Histoire et de la civilisation marocaines : «Depuis que j'étais au Maroc, j'avais la sensation d'être devant un peuple, un vrai peuple, développé par sa civilisation propre, ayant derrière soi les siècles d'un peuple ». (André Chevrillon, 2008 : 97)

7 Parler d'un «vrai peuple» et d'« une civilisation propre» est une manifeste reconnaissance de la pluralité des cultures dont chacune a des spécificités et des particularités qui sont censées être comprises sans référence à des modèles extérieurs. Après avoir mis son pied en Italie, Michel de Montaigne n'a-t-il pas dit : "J'ai quitté un monde et je suis dans un autre»? $(1994,31)$.

Chevrillon est d'une culture anglaise plus encline à accepter la différence. Sa relation de voyage peut être considérée comme un voyage initiatique qui lui permet de "sculpter son moi » (Onfray, 1993) au contact des réalités marocaines. A travers un regard esthétique qu'il porte sur le Maroc, il va se forger une morale qui l'incite au respect de l'autre. En effet, il vit pleinement son expérience de rencontre avec ce monde autre, rejette toute prétention à la normativité et reconnaît même, ce qui est 
d'ailleurs très rare chez les orientalistes, la contribution du monde arabo-musulman à l'évolution historique de l'Europe :

$\mathrm{Au}$ premier feu des compliments adressés en ma personne à la science de la chrétienté, nous avons riposté par une allusion à leurs ancêtres, les Arabes d'Espagne qui ramenèrent la science dans l'Europe du Moyen-âge. (André Chevrillon, $2008: 213$ )

9 Cette partie du dialogue entre le sultan du Maroc et André Chevrillon pourrait être considérée comme une plate-forme de reconnaissance et de réciprocité entre l'Orient et l'Occident. En vérité, chacun de ces deux pôles est appelé à contribuer de son côté à la dynamisation et la progression de l'humanité. Celle-ci ne pourrait avancer qu'à travers la fécondité des différences humaines et le respect de la variété des systèmes de pensée. Une telle perspective implique explicitement la vision égalitaire des cultures condamnées à cohabiter et à coexister.

Par ailleurs, le voyage au Maroc n'est pas seulement pour le voyageur un moment d'échange, de réciprocité et d'interculturalité, c'est aussi une occasion de réflexion sur soi-même ${ }^{5}$ et de remise en cause de sa culture d'appartenance ${ }^{6}$. Dans ce contexte, l'expérience existentielle d'André Chevrillon est primordialement orientée par une consciente tendance vers l'introspection autant que vers la rétrospection. En effet, comprendre et connaître l'altérité permet au voyageur de mieux se comprendre et de mieux se connaître. Le contact avec le monde autre procure chez lui de nouvelles sensations et surtout lui révèle les insuffisances de la civilisation occidentale qui, de son point de vue, "sombre dans l'artificialité" (André Chevrillon, 2000: 46). Ce regard critique inclut un rejet de l'hégémonisme occidental que Georges Corm appelle "l'autosatisfaction narcissique du discours véhiculé en Occident sur les prodiges de sa civilisation » (Georges Corm, 2009 : 46).

Le relativisme culturel constitue un antidote à cette prétention à la perfection et à l'exemplarité du modèle, dit hégémonique, occidental. Ce modèle, imprégné d'ethnocentrisme, est impropre à la formation d'un monde multiculturel et multipolaire. L'Ailleurs développe chez Chevrillon une attitude morale et culturelle fondée essentiellement sur la reconnaissance et le respect de l'Autre dans son altérité, sa différence et sa particularité. Par cette réflexion altruiste, à dimension relativiste ${ }^{7}$, le voyageur, croyant à un monde au pluriel cherche, dans le rapport avec l'altérité, "la possibilité d'une différence» (Roland Barthes, 2002: 351). Aussi, rejette-t-il catégoriquement l'uniformisation du monde à partir d'un seul contexte civilisationnel et d'un seul modèle culturel. De fait, c'est en confrontant les systèmes les plus éloignés et en instaurant une correspondance entre les peuples les plus dissemblables que l'on s'assure de la relativité de ce que l'on a cru vrai et naturel. En outre, on s'aperçoit de la force de la solidité des modèles culturels autres. Le relativisme culturel est ainsi une attitude positive permettant une vision claire et une ouverture sur des pensées multiples ; ce qui a pour effet la création d'un cadre de respect et de reconnaissance de toutes les civilisations qui se valent et s'équivalent sans hiérarchisation ni stratification. Un égalitarisme pareil contredit au fond l'idée de l'universalisme qui prône la globalisation de la pensée unique. 


\section{L'exotisme chez chevrillon : un éloge de la différence}

12 L'exotisme littéraire, forge des symboles culturels sous lesquels l'ailleurs et l'altérité ne sont perçus et conçus qu'à travers le prisme de l'inhabituel et de l'étrangeté: "L'exotisme est une conception toute faite que nous avons d'un pays et de ses habitants" (Pierre Jourda, $1970: 15$ ).

13 Il s'agit d'une conception réductrice qui fortifie le processus de hiérarchisation des cultures et des civilisations. Cette hiérarchisation reflète la tendance de l'Occident à se positionner en tant que centre éthique, esthétique, culturel et civilisationnel du monde. Cette posture hégémonique entraîne une tentative d' "exotisation» de ce qui n'appartient pas à la sphère occidentale. Cela dit, l'exotisme dans sa signification traditionnellement essentialiste, comme le perçoit par exemple Pierre Loti ${ }^{8}$, se résume à une attitude culturaliste qui tente de tracer des frontières et des distances symboliques entre l'Occident et le reste du monde. Cet "exotisme de bazar" (Victor Segalen, 1978 : 31), comme préfère l'appeler Segalen, est loin d'être une expression d'admiration, mais c'est une infériorisation de ce qui échappe au « déjà vu » et au « déjà connu » et de ce qui est qualifié d'insolite, de bizarre et de non naturel. Chevrillon a tenté de faire le contrepoids de ce genre d'exotisme lotien. De ce fait, il s'est employé à médiatiser une expérience exotique qui s'élève à une profonde quête du "Divers ». Pour lui, appréhender le monde lointain c'est essentiellement l'intérioriser pour accéder au contact fructueux avec la diversité. Cette poétique de la diversité est un remède contre la simple folklorisation et infériorisation de l'ailleurs, une poétique indissociable d'une éthique qui permettrait de sentir, de connaître et reconnaître la différence loin de tout regard réducteur ou destructeur.

Dans ce sillage, le voyageur français a rompu avec l'approche fantasmatique fondée sur la quête du merveilleux et sur le triomphe de l'étrangeté au détriment de l'extériorité dans son originalité et sa vérité. Pour Jean-Marc Moura : "L'exotisme qui commence avec l'attention portée à la réalité étrangère, disparait donc souvent derrière les figures d'une imagination portée sur le merveilleux» (1992: 44).

Contrairement à cette " imagination portée sur le merveilleux », Chevrillon a opté pour un exotisme qui s'apparente à une perception intimiste du monde et à un élan vers l'altérité dans sa multitude et sa diversité. Chevrillon, pour paraphraser Segalen, déguste le divers et adopte l'exotisme dans sa signification profonde ${ }^{9}$ qui est cette prise de conscience de ce qui n'est pas soi-même et de ce qui est différent de soi. Il opte pour un exotisme qui exhibe l'humanité dans sa vérité et sa variété. Son exotisme est primordialement une quête humaniste et abyssale et non une quête simplificatrice et ornementale transfigurant l'étrangeté et l'étranger en de simples objets décoratifs et attractifs. Sur ce plan, le voyageur français a vécu son expérience exotique au Maroc en célébrant la diversité synonyme d'équilibre et de vitalité. Reconnaître cette diversité c'est s'opposer à la logique unique, homogène et totalitaire. C'est une reconnaissance qui ouvre la voie à la connaissance. Il s'engage dans un processus de découverte de nouvelles formes idéales et culturelles, des découvertes marquées par l'exaltation du beau et par la mise en scène de la sublimité du divers: "Les bédouins qui vendent leurs herbes et leurs oignons dans le creux de la rue, sont virilement beaux» (André Chevrillon, $2000: 33)$. 

voyageur porte sur les êtres et les objets qu'il rencontre et qu'il décrit. Ce regard se focalise sur la part de sublime que chaque élément détient. Ce qui intéresse le voyageur, c'est "l'esthétique du divers » (Victor Segalen, 1978 : 96), qui consiste à admirer la différence pour sa beauté et sa richesse et non pas pour son étrangeté et son anormalité attractive. Sans cette différence, la vie humaine serait condamnée à la monochromie, à la fadeur et à la ressemblance. Et cette ressemblance génère systématiquement l'insignifiance. Conscient ainsi de l'importance de la diversité et du dynamisme de la pluralité, Chevrillon se réjouit du «divers ", une réjouissance qui se procure loin de toute attitude méprisante de l'étrangeté et de la particularité comme en témoigne le passage suivant :

Grave décor d'une richesse toute abstraite : nulle image du monde réel ne vient s'y mêler pour occuper l'esprit. Sous ces prestiges de la pure couleur qui chante sensuellement et s'harmonise dans l'ombre, la pensée reste plus facilement oisive, sans effort, le regard suit les alternances rouges et jaunes de ces ogives répétées qui ne sont que mythe et musique sur les murs. On se laisse pénétrer par ces influences; elles engourdissent comme une vapeur de haschich : c'est dans leurs demeures que ces Maures apprennent les délices de se taire, et devant un samovar et de petites tasses, de se muer en choses. (André Chevrillon, 2000 :108-109)

Cette propension à se laisser influencer ${ }^{10}$ par l'ailleurs fait preuve de la dimension fructueuse de l'exotisme tel que le voyageur le conçoit. Ce dernier manifeste aussi son adaptabilité avec la culture autre qui fortement l'impressionne. L'étrangeté, dans ce contexte, impacte positivement et productivement le comportement, la pensée, les sentiments et les sensations de l'auteur qui sort de sa coquille occidentale pour se familiariser avec l'univers lointain. Dès lors, le contact avec l'étrangeté devient, pour lui, un moyen d'élargissement des horizons culturels ${ }^{11}$ et un moment de remise en question de sa propre perception du monde.

Il s'avère que le voyageur ne cherche pas au Maroc le fantastique ou le sentimental à travers une affinité avec des objets ou des êtres « exotiques ». En revanche, il porte un intérêt considérable à la réalité marocaine décrite en toute objectivité dans le but d'en rapporter l'image de la façon la plus claire et la plus réelle possible :

$\mathrm{Au}$ dehors, sous les étoiles naissantes, le grand terrain de campement fourmille encore ; les moutons bêlent et s'agenouillent par troupeaux dans la poussière, les ânes et les mulets entravés sont au piquet, les chameaux par terre tirent l'herbe de leur tas commun de fourrage, ils mâchent en somnolant. A travers la foule vont et viennent les sorciers nègres, coiffés de leurs tiares de coquillages, battant du tambourin, au passage, ils nous jettent d'affables grimaces. (Ibid. : 212)

Ce spectacle authentique et exotique mettant en scène des objets atypiques évoque un monde autre que celui du voyageur. Celui-ci insiste sur ce qui le frappe en profondeur dans cet univers qu'il traite et interprète avec respect et sensibilité sans en diminuer les potentialités et sans lui témoigner du mépris. L'une de ces potentialités qui distinguent le Maroc précolonial est sa musique "exotique », en l'occurrence la musique Andalouse ${ }^{12}$ dont Chevrillon ne cesse d'exalter et de mettre en valeur les qualités essentielles :

C'est la plus belle des musiques! Si belle qu'on n'en cherche plus d'autre... On ne pourrait pas faire si bien. Mais les paroles! Les métaphores! C'est là qu'est la plus admirable beauté. Quels maîtres, ces poètes andalous de la langue littéraire! Il faut avoir étudié longtemps pour sonder cela, pénétrer les sens multiples, bien deviner ce qui n'est pas dit. Ecoute: maintenant ils chantent que la bien-aimée est un échanson. Dans l'orgie, à la cour de l'émir, cet échanson présente un cratère de vin. 
Le poème donne le nom de ce vin. Eh bien! pour celui qui voit, ce nom veut dire aussi la salive de la bien-aimée. Quelle profondeur! Et voici un autre mot qui désigne à la fois le vin qui est rouge, la lèvre de la bien-aimée qui est rouge, sa pommette qui est rouge ! (Ibid. : 243-244).

La musique Andalouse, en tant que figure de la culture et de la beauté artistique du Maroc, fascine considérablement l'écrivain voyageur aussi bien par son rythme, sa rhétorique et sa poéticité. Par ce goût pour l'exotisme musical, Chevrillon rapporte une version esthétique de la réalité marocaine. Une réalité décrite dans son objectivité et son essence et non pas dans sa subjectivité et sa surface. L'esthétique de Chevrillon s'inscrit ainsi dans le cadre d'une configuration de pensée héritée de ce qu'on appelle « le mal du siècle ». Elle a pris acte de l'irrémédiable processus du désenchantement du monde qui a affecté l'Europe à partir de la Renaissance. L'art devient ainsi cette alchimie qui consiste à rendre présent ce qui a été perdu à jamais à cause de la modernité. Cette alchimie consiste en la déréalisation de l'espace marocain. Ce faisant, le voyageur fait preuve de sa capacité à sentir et à percevoir le monde étranger en termes de sensibilité et de sublimité plutôt qu'en termes de banalité et de folklorisation. Autrement dit, Chevrillon opte pour l'esthétisation de l'altérité et de l'ailleurs plutôt que pour leur « exotisation ».

21 A la fin, nous constatons qu'aux antipodes des écrivains voyageurs qui cherchent à puiser dans l'exotisme des images superficielles, André Chevrillon vit de l'intérieur et avec profondeur son expérience de voyage. En effet, il avance vers l'inconnu, cette source d'énergie, qui lui permet de s'ouvrir sur l'extérieur pour s'en remettre à son être intérieur. Le neveu d'Hippolyte Taine a donc pu développer une éthique de relation, de proximité et d'intimité avec l'ailleurs et l'altérité, une relation fondée fondamentalement sur le respect, le partage, la connaissance et la reconnaissance. Sur ce plan, Un crépuscule d'Islam propose de nouvelles pistes de visibilité et de lisibilité de l'altérité. Elle invite aussi à renouveler les perspectives d'ouverture sur l'Autre au-delà des étiquettes globalisantes et des représentations stigmatisantes. Son auteur, loin des mécanismes idéologiques, s'emploie, tout au long de son voyage, à réserver au Marocain une place qui ménage à la fois sa singularité, sa typicité et sa spécificité. C'est dire que cette expérience marocaine est orientée vers ce qu'Edouard Glissant (1996) appelle "la poétique du divers", une poétique fondée sur la reconnaissance de la diversité et la prise de conscience de la pluralité des identités. Un tel relativisme culturel constitue une disqualification du réductionnisme et de l'ethnocentrisme qui tendent à légitimer la catégorisation, la hiérarchisation et la discrimination. De fait, c'est contre tout discours classificatoire ou discriminatoire que le voyageur français forge une approche constructiviste où il élargit sa réflexion à l'interculturalité, contexte privilégié pour le contact et la réciprocité. Plus qu'un moment de cloisonnement des cultures, le voyage de Chevrillon au Maroc est une expérience exotique, c'est-à-dire une expérience de l'étrangeté, de la curiosité et de l'hybridité. Il n'en demeure pas moins que l'exotisme sous sa plume, n'est pas un acte de puissance sur autrui, mais c'est un travail introspectif et une réflexion ontologique sur l'intériorité dans son rapport dialectique avec l'extériorité. En effet, sans qu'il soit une fascination pour la surface de cette extériorité, l'exotisme chez Chevrillon dépasse la vision substantialiste pour renvoyer à une perception intimiste de la réalité dans sa complexité et son hétérogénéité. L'écrivain voyageur s'est démarqué ainsi de la vision orientaliste et ethnocentriste qui réduit l'altérité à la négativité et à l'infériorité. Il a réussi, en parallèle, à développer un discours novateur qui perçoit la différence en 
termes d'ouverture, d'interpénétration et d'intercompréhension et non pas en ceux de fixation, d'exclusion et de stigmatisation.

\section{BIBLIOGRAPHIE}

BARTHES, Roland, L'empire des signes, Euvres complètes, Editions du Seuil, Paris, 2002.

CHEVRILLON, André, Un crépuscule d'Islam, Editions EDDIF, Casablanca, 2008.

CORM, Georges, L'Europe et le mythe de l'Occident, Editions La Découverte, Paris, 2009.

GLISSANT, Edouard, Introduction à une poétique du Divers, Editions Gallimard, Paris, 1996.

HUNTINGTON, Samuel, Le choc des civilisations, Traduit de l'anglais par Jean-Luc Fidel, Editions Odile Jacob, Paris, 2000.

JOURDA, Pierre, L'exotisme dans la littérature française, Editions Slaktine, Genève, 1970.

LEVINAS, Emmanuel, Autrement qu'être ou Au-delà de l'essence, Editions Verdier, Paris, 1984.

MALHERBE, Michel, Les cultures de l'humanité, Editions du Rocher, Paris, 2000.

MONTAIGNE, Michel, Essais II, Editions Flammarion, Paris, 1994.

MOURA, Jean-Marc, Lire l'exotisme, Editions Dumond, Paris, 1992.

ONFRAY, Michel, La sculpture de soi, La morale esthétique. Editions Biblio. Essai, Le livre de poche, 1993.

SAÏD, Edward, L'orientalisme, L'Orient créé par l'Occident, traduit de l'américain par Catherine Molammoud, Editions du Seuil, Paris, 1980.

SARTRE, Jean-Paul, L'Être et le néant, Editions Gallimard, Paris, 1976.

SEGALEN, Victor, Essai sur l'exotisme, Editions Fata Morgana, Paris, 1978.

SEGALEN, Victor, Brique et tuiles, Editions Fata Morgana, Paris, 1980.

TODOROV, Tzvetan, Nous et les autres, La réflexion française sur la diversité humaine, Editions du Seuil, Paris, 1983.

\section{NOTES}

1. Chez Jean-Paul Sartre, le rapport à l'autre prend souvent la forme de l'affrontement et de la confrontation: "Pendant que je tente de me libérer de l'espace d'autrui, autrui tente de se libérer du mien. "L'Être et le néant, Ed. Gallimard, Paris, 1976: p.389. Chevrillon semble dépasser cette polarité dialectique et cette dimension conflictuelle. Sa relation avec l'autre, en l'occurrence la Marocain, pourrait être placée sous le signe de la compréhension et de la sympathie. Cette approche d'ouverture est aussi adoptée par Levinas qui appelle à écouter et à regarder l'autre, à le rencontrer et à l'appréhender dans son altérité et sa diversité : "Seul le sens d'autrui est irrécusable et interdit la réclusion et la rentrée dans la coquille du soi. Une voix vient de l'autre rive. Une 
voix interrompt le dire du déjà dit " écrit-il. (Emmanuel Levinas, Autrement qu'être, Au-delà de l'essence, op.cit., p.23.)

2. Pour plus de précisions sur la vision dichotomique entre le Moi et l'Autre, voir La peur des barbares, au-delà du choc des civilisations, Tzvetan Todorov, Editions Robert Laffont, Paris, 2008.

3. Les relations entre l'Orient et l'Occident sont encore, à notre époque, marquées par l'ignorance mutuelle et par la méconnaissance profonde des traits majeurs de chacun de ces deux pôles civilisationnels. Ces relations sont surtout affectées par des préjugés hérités du passé. C'est ce qui nous amène à nous interroger sur la nature du choc entre l'Est et l'Ouest. Est-il un «choc des civilisations ", pour reprendre l'expression de Sammuel Huntington, ou simplement un choc des imaginaires?

4. Le relativisme culturel est une interprétation du rapprochement et de la reconnaissance des formes et des modèles culturels divers, loin de toute idée de centralité, de normativité et de conformité.

5. Le voyage de Chevrillon est déterminé par la volonté de rompre avec sa culture occidentale et le désir d'échapper à une civilisation désenchantante et décevante.

6. Ce fléchissement du regard consiste à passer de la réflexion sur l'extériorité à la découverte de la réalité et la vérité de l'intériorité. Todorov qualifie cette démarche en termes de : «Découvrir le propre par le différent ", Nous et les autres, Editions du Seuil, Paris, 1983 : p.43.

7. Si le relativiste croit à l'arbitraire culturel, c'est-à-dire à chaque peuple sa culture et à chaque culture ses propres valeurs, l'ethnocentriste, en revanche, érige ses valeurs en vérité absolue et en normes universelles.

8. Pierre Loti reste l'un des précurseurs de l'exotisme dans sa signification classique, c'est-à-dire un exotisme représentatif d'une vision fantasmée de l'altérité et de l'ailleurs perçus à travers le prisme de l'étrangeté caricaturale. Segalen a été catégorique dans sa critique de Pierre Loti. Il a même dédaigneusement usé du néologisme "Lotiforme " pour dénoter négativement la forme de l'exotisme lotien : "Dans cette chaine de quelques vers, de quelques musiques parée de tant de durée, de tant d'équivoque, de tant de préciosité sauvage et de frugalité... Mais ceci combien Lotiforme. » Brique et tuiles(1909), Ed .Fata Morgana, Montpelier, 1980, p. 38.

9. L'exotisme, tel qu'il est conçu par Segalen, correspond à une fascination de l'Autre. Ce qui donne lieu à une réelle et profonde expérience de l'Altérité : «L'exotisme n'est autre que la notion du différent, la perception du Divers; la connaissance que quelqu'un n'est pas soi-même; et le pouvoir d'exotisme, qui n'est que le pouvoir de concevoir l'autre. » Essais sur l'exotisme, op.cit., p.36.

10. La découverte de l'Autre exotique conduirait en même temps à la redécouverte de soi-même. Cette productivité de la proximité ne veut pas dire une dissolution dans l'altérité ou une rupture avec l'identité. Tzvetan Todorov confirme : "Pour éprouver l'Autre on n'a pas besoin de cesser d'être soi. » Nous et les autres, Editions du Seuil, Paris, 1983 : p.407.

11. Au lieu d'exprimer une dualité culturelle, l'exotisme peut motiver un rapprochement entre des aires civilisationnelles et géographiques hétérogènes.

12. La musique Andalouse est un genre musical savant qui a été amené au Maroc après la chute de Grenade. Ce genre musical s'est établi notamment à Fès, Tétouan, Rabat et Oujda. 


\section{RÉSUMÉS}

André Chevrillon, lors de son voyage au Maroc, a pu faire de la rencontre avec l'altérité un moment d'échange culturel où la diversité et le pluralisme sont largement mis en avant. Cette rencontre est aussi un moment de réflexion sur l'Autre et sur soi, une réflexion qui se cristallise par la reconnaissance de la validité de l'existence des systèmes de pensée autres que ceux de l'Occident. L'écrivain voyageur s'est démarqué ainsi de l'exotisme classique et de la vision orientaliste et ethnocentriste qui réduit l'altérité à la négativité et à l'infériorité. Il a réussi, en parallèle, à développer un discours novateur qui perçoit la différence en termes d'ouverture, d'interpénétration et d'intercompréhension et non pas en ceux de fixation, d'exclusion et de stigmatisation.

During his trip to Morocco, André Chevrillon experienced alterity, a phase of cultural exchange, characterized by diversity and pluralism. This experience is also a phase of reflection on the Self and the Other. Such reflection is characterized by the acknowledgement of the validity of the existence of thought patterns that are different from those of the West. Therefore, the travel writer distinguished himself from classic exosticism and from an orientalist ethnocentrist vision that minimises alterity to negativity and inferiority. In parallel, he also succeded in developing an innovative discourse that perceives difference in terms of openness, interpenetration, and mutual understanding, not of fixation, exclusion, and stigmatization.

\section{INDEX}

Mots-clés : voyage, altérité, relativisme, exotisme, interculturalité

$$
\text { رحله الغيرية النسبية, الغرائبية, التفاعل الثقافيفهرس الكلمات المفتاحية: }
$$

Keywords : travel, otherness, relativism, exotism, interculturality

\section{AUTEUR}

\section{ABDELHAK ZERRAD}

Université Sidi Mohamed Ben Abdallah

Fès 